\title{
The axis of evil - a polarization perspective
}

\section{Mona Frommert*}

Max-Planck-Institut für Astrophysik, Garching, Germany

E-mail: mona@mpa-garching.mpg.de

\section{Torsten A. Enßlin}

Max-Planck-Institut für Astrophysik, Garching, Germany

E-mail: ensslin@mpa-garching.mpg.de

\begin{abstract}
We search for an unusual alignment of the preferred axes of the quadrupole and octopole, the so-called axis of evil, in the WMAP polarization data. We split the polarization map into a part correlated with the temperature and an uncorrelated part. We then determine the preferred axes for the quadrupole and octopole in the uncorrelated polarization map, which serves as a statistically independent probe of the axis of evil. We find that the axis of the quadrupole aligns with the axis of evil within our measurement precision, whereas the axis of the octopole does not. However, with our measurement uncertainty, the probability of at least one axis aligning by chance in an isotropic universe is of the order of 50 per cent. Nevertheless, our analysis of the axis of evil promises to be a powerful tool when applied to data expected from the Planck satellite.
\end{abstract}

International Workshop on Cosmic Structure and Evolution - Cosmology2009, September 23-25, 2009

Bielefeld, Germany

\footnotetext{
* Speaker.
} 


\section{Introduction}

The observed isotropy of the cosmic microwave background (CMB) is one of the strongest evidences for the isotropy of the Universe. However, in recent years, there have been claims of anomalies detected in the CMB temperature map with considerable significance, which seem to break statistical isotropy of the temperature fluctuations and thus to question the cosmological principle. Among these anomalies, there is a strong alignment between the preferred axes of the quadrupole and the octopole, which is commonly referred to as the axis of evil $[3,1,11,16,15]$. The claims of the existence of a preferred direction in the CMB temperature map have led to a discussion about whether this is simply due to a chance fluctuation in the CMB temperature map, if it can be blamed on local structures or on systematics in the measurement, or whether it is actually due to a preferred direction intrinsic to the geometry of the primordial Universe $[4,12,17,8,13$, $18,10]$.

Since the polarization fluctuations of the CMB have the same physical origin as the primordial temperature fluctuations, they should exhibit similar peculiarities as the latter, if these are intrinsic to the geometry of the primordial Universe. ${ }^{1}$ However, since the polarization is not statistically independent of the temperature, chance fluctuations in the temperature map also manifest themselves in the polarization [6]. Here, we thus use the part of the polarization map which is uncorrelated with the temperature map as a statistically independent probe of the axis of evil. Chance fluctuations in the temperature maps do not affect the uncorrelated polarization map, so that a detection of the anomalies in the latter would be a hint to an actual cosmological origin of them.

\section{The axis of evil in temperature}

We start by searching for the axis of evil in the CMB temperature map. To this end, we use the internal linear combination (ILC) map from WMAP smoothed with a kernel of $18.3^{\circ}$. When working on large scales, we can safely neglect the detector noise in the temperature data [2], and we decide to neglect residual foregrounds in the temperature map as well.

To define the preferred axis, we use the statistic proposed by de Oliveira-Costa et al. [3], which has been introduced in order to quantify the preferred direction that can be picked out in the smoothed temperature map by eye. When looking at the smoothed ILC map in Fig. 1, most of the hot and cold blobs seem to be lying on the same plane. The quadrupole and octopole extracted from the ILC map show the same behaviour [see, e.g., 3], and the planes are roughly the same for the two multipoles. In order to quantify this alignment, de Oliveira-Costa et al. came up with the following statistic. The temperature maps are expanded into spherical harmonics, which are eigenfunctions of the square and the z-component of the angular momentum operator $L$ :

$$
T(\hat{n})=\sum_{l} T_{l}(\hat{n}) \equiv \sum_{l, m} a_{l m}^{T} Y_{l m}(\hat{n})
$$

\footnotetext{
${ }^{1}$ Note, though, that this is not generic for all models exibiting anomalies in the temperature map. For example, if the peculiarities in the temperature maps are due to a secondary effect on the CMB such as the integrated Sachs-Wolfe effect, we would not expect them to be present in the polarization maps [6].
} 


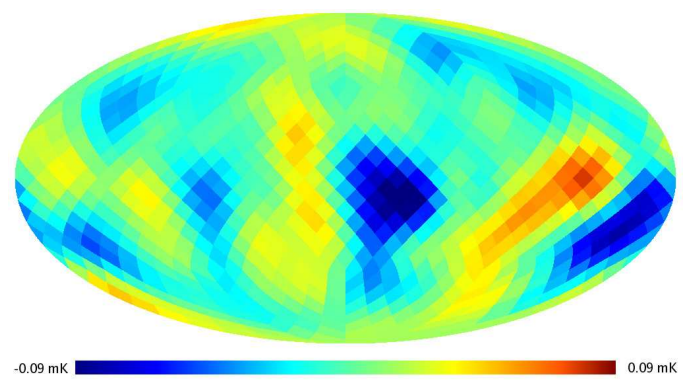

Figure 1: ILC map, smoothed with a beam of $18.3^{\circ}$ and downgraded to a resolution of NSIDE $=8$.
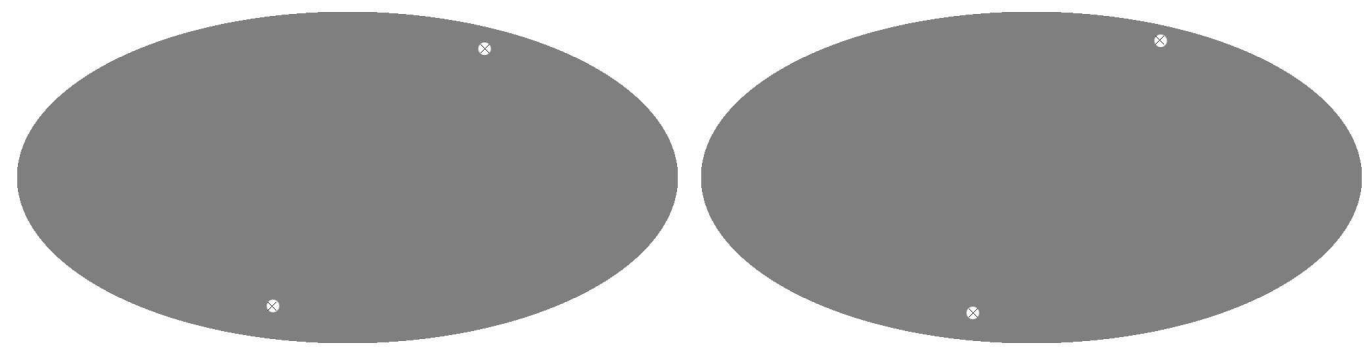

Figure 2: Preferred axis of the quadrupole (left panel) and the octopole (right panel) for the ILC map. We reproduce the results of de Oliveira-Costa et al. within our measurement precision. The axes of quadrupole and octopole point in the same direction, which has been named the axis of evil.

Then, for every multipole $l$, we determine the z-axis $\hat{n}$ for which the expectation value of the $\mathrm{z}$ component of $L, \hat{n} \cdot L$, is maximised:

$$
\hat{n} \equiv \max _{\hat{n}^{\prime}}\left\langle\left. T_{l}|| \hat{n}^{\prime} \cdot L\right|^{2} \mid T_{l}\right\rangle=\sum_{m} m^{2}\left|a_{l m}\left(\hat{n}^{\prime}\right)\right|^{2}
$$

Here, $a_{l m}\left(\hat{n}^{\prime}\right)$ denotes the spherical hamonic coefficient $a_{l m}$ obtained in a coordinate system with the z-axis pointing in $\hat{n}^{\prime}$-direction. We determine the axis $\hat{n}$ by simply rotating the z-axis into every pixel centre and checking for the maximum, which is well feasible at our resolution.

We plot the preferred axes of the quadrupole and the octopole of the ILC map in Fig. 2. Both ends of every axis are marked by a cross in the maps. We reproduce the results from de OliveiraCosta et al. within our measurement precision: the axes of the quadrupole and the octopole of $P_{\text {corr }}$ point in the same direction, which is roughly $(l, b) \approx\left(-120^{\circ}, 63^{\circ}\right)$, where $l$ and $b$ denote galactic longitude and latitude, respectively (de Oliveira-Costa et al. find $(l, b) \approx\left(-110^{\circ}, 60^{\circ}\right)$ ).

\section{Splitting of the polarization map}

Let us now move on and obtain the uncorrelated polarization map, in which we are then going to search for the axis of evil. Unfortunately, the WMAP polarization data are highly contaminated by detector noise and galactic foregrounds. Our observed polarization map, $P$, is the linear combination of the maps of the Ka, Q, and V frequency bands (corresponding to 33, 41, and $61 \mathrm{GHz}$ ), which are used for determining the low-l polarization likelihood in the 5 year WMAP likelihood code [9]. We furthermore use the P06 mask [14] to mask out the galactic plane in the polarization 


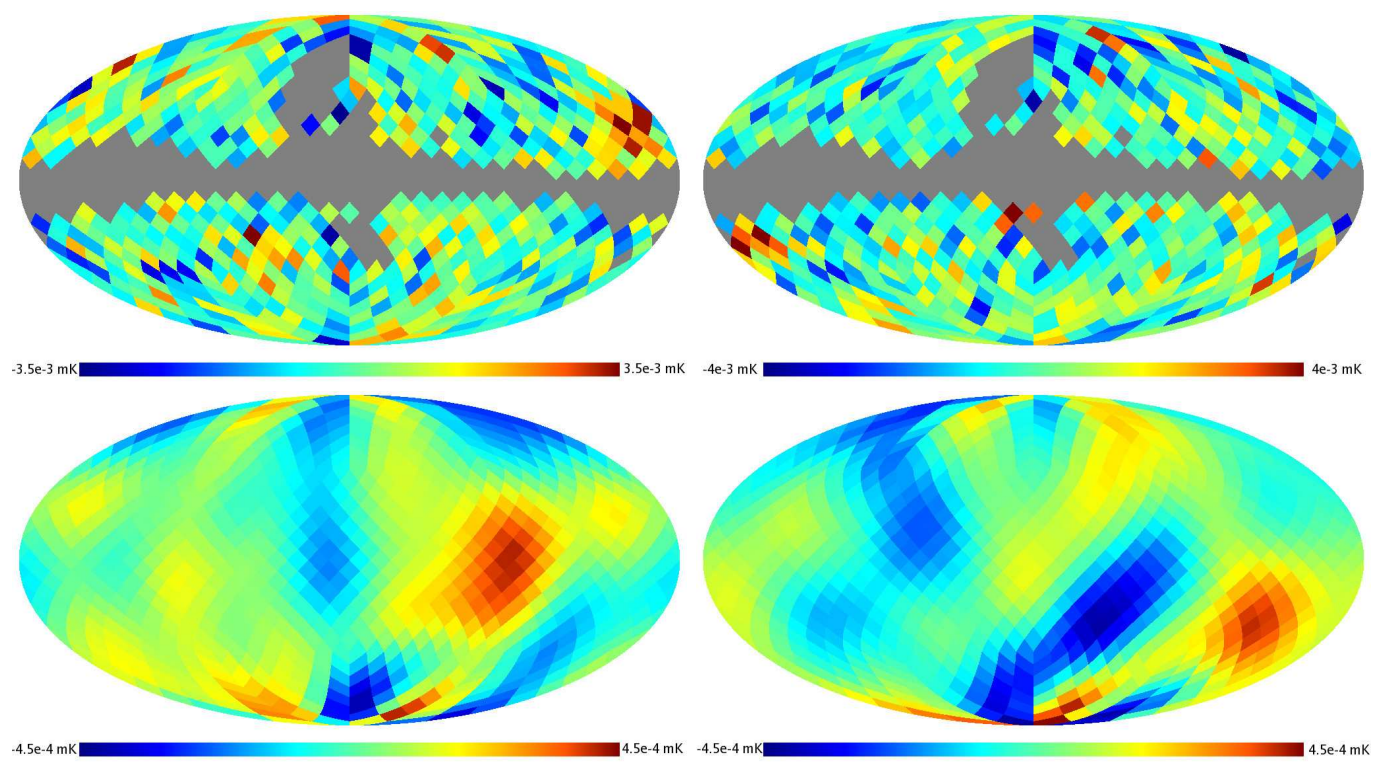

Figure 3: Stokes Q (left) and U (right) part of the following polarization maps: Top panel: Observed polarization maps (linear combination of $\mathrm{Ka}, \mathrm{Q}$, and $\mathrm{V}$ band maps). Bottom panel: Part of the polarization map uncorrelated with the temperature map, $P_{\text {uncorr }}^{\text {rec }}$.

map. The Stokes $\mathrm{Q}$ and $\mathrm{U}$ parameters for $P$ in galactic coordinates are shown in the top panel of Fig. 3.

We now split the WMAP polarization map into a part correlated with the WMAP temperature map, $P_{\text {corr }}$, and a part uncorrelated with that, $P_{\text {uncorr }}^{\text {rec }}$. We obtain the correlated polarization map by simply translating the temperature map into a polarization map:

$$
P_{\text {corr }} \equiv S_{P, T} S_{T}^{-1} T
$$

where the signal covariance matrices are defined as $S_{P, T} \equiv\left\langle P_{\mathrm{cmb}} T^{\dagger}\right\rangle$ and $S_{T} \equiv\left\langle T T^{\dagger}\right\rangle$, with $P_{\mathrm{cmb}}$ being the noiseless CMB-polarization map. In order to obtain the uncorrelated map, we would like to subtract $P_{\text {corr }}$ from $P_{\mathrm{cmb}}$ :

$$
P_{\text {uncorr }} \equiv P_{\text {cmb }}-P_{\text {corr }} \text {. }
$$

However, we do not know $P_{\mathrm{cmb}}$ because we only observe $P$, which is highly contaminated by noise. We therefore subtract $W P_{\text {corr }}$ from the observed polarization map $P$ :

$$
P_{\text {uncorr }}^{\text {raw }} \equiv P-W P_{\text {corr }},
$$

where $W$ denotes the sky mask. We then perform a Wiener filtering on $P_{\text {uncorr }}^{\text {raw }}$ in order to obtain the noise-reduced uncorrelated polarization map $P_{\text {uncorr }}^{\text {rec }}$. Note that the index "rec" indicates that the map we obtain is a Wiener reconstruction of $P_{\text {uncorr }}$. We show the Stokes Q and U maps of the uncorrelated polarization map, $P_{\text {uncorr }}^{\text {rec }}$, in the bottom panel of Fig. 3 .

\section{The axis of evil in the uncorrelated polarization map}

We now determine the preferred axes of the quadrupole and the octopole for the uncorrelated polarization map, again using the technique introduced in section 2 . As we have already mentioned, 

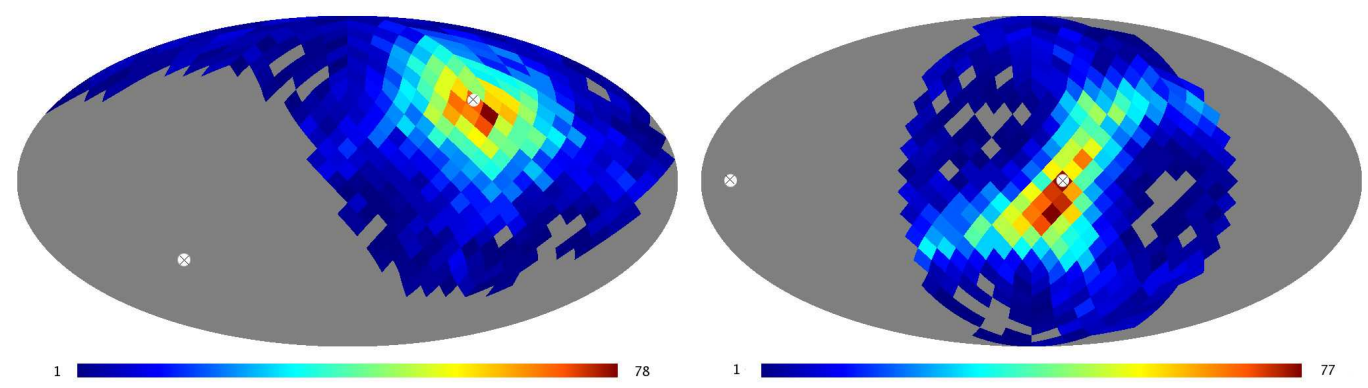

Figure 4: Preferred axis of the quadrupole (left panel) and the octopole (right panel) for $P_{\text {uncorr }}^{\text {rec }}$. The colour coding counts the number of MC samples whose axis came to lie on the respective pixel. The axis of the quadrupole aligns with the axis of evil within our measurement precision, whereas the axis of the octopole does not.

the mask, residual foregrounds and detector noise in the polarization data will result in an uncertainty in the preferred axes. In order to obtain the uncertainties in the axes of $P_{\text {uncorr }}^{\text {rec }}$, we have run Monte Carlo (MC) simulations, drawing realisations of $P_{\text {uncorr }}$ conditional on the measured data, for which we then determine the preferred axes. We plot the axes and their uncertainties for $P_{\text {uncorr }}^{\text {rec }}$ in Fig. 4. Again, both ends of every axis are marked by a cross in the maps, and the colour coding counts how many times the preferred axis came to lie on the respective pixels in $5000 \mathrm{MC}$ samples. The axis of the quadrupole points to $(l, b)=\left(-79^{\circ}, 36^{\circ}\right)$ with an uncertainty of $42^{\circ}$, and the one of the octopole to $(l, b)=\left(-17^{\circ}, 0^{\circ}\right)$ with an uncertainty of $48^{\circ}$. The axis of evil lies inside the $1 \sigma$ region of the quadrupole, but not of the octopole of $P_{\text {uncorr }}^{\text {rec }}$.

What can we learn from this result? The significance of the alignment between the axes of the quadrupole and octopole in the temperature map has been assessed extensively in earlier works. In this work, we only look at the additional information we obtain from the axes of $P_{\text {uncorr }}^{\text {rec }}$ To this end, let us take the preferred axis in the temperature map $T$ as given, and assume that the axes of $P_{\text {uncorr }}^{\text {rec }}$ are distributed isotropically over the sky and independently of each other. The probability for at least one of the axes of $P_{\text {uncorr }}^{\text {rec }}$ aligning with the axis of evil in an isotropic Universe amounts to about 50 per cent, due to the large uncertainties we have. We therefore do not obtain evidence for or against a preferred direction intrinsic to the geometry of the primordial Universe from $P_{\text {uncorr }}^{\text {rec }}$ Nevertheless, a very crude estimate for Planck yields an uncertainty in the axes of about $10^{\circ}-20^{\circ}$. With this, we will have a powerful test to probe the axis of evil in polarization.

\section{Conclusions}

We have computed the part of the WMAP polarization map which is uncorrelated with the temperature map, $P_{\text {uncorr }}^{\text {rec }}$, and used it as a statistically independent probe of the axis of evil. If the axis of evil were due to some preferred direction intrinsic to the geometry of the primordial Universe, we would expect its signature to be present in $P_{\text {uncorr }}^{\text {rec }}$, whereas we would not expect to see it in $P_{\text {uncorr }}^{\text {rec }}$ if it were due to chance fluctuations in the temperature. In order to reduce the noise contained in the polarization maps, we have Wiener filtered the uncorrelated polarization map before computing the axes. We have then determined the preferred axes of the quadrupole and the 
octopole for $P_{\text {uncorr }}^{\text {rec }}$. In order to assess the uncertainty in the axes coming from the mask, detector noise and residual foregrounds, we have run MC simulations conditional on the observational data.

We find that only the axis of the quadrupole aligns with the axis of evil, whereas the axis of the octopole does not. We have computed the probability that a rough alignment with the axis of evil for at least one of the axes happens by chance if the axes are distributed isotropically. This probability amounts to 50 per cent for currently available polarization data, due to the large uncertainties in the axes. We thus do not obtain evidence for or against a preferred direction intrinsic to the primordial Universe from WMAP, but are looking forward to redoing this analysis with polarization maps from Planck, which will yield much more significant results.

\section{Acknowledgements}

The authors would like to thank Simon White for a suggestion that greatly improved this work. We further thank Thomas Riller, Carlos Hernandez-Monteagudo, Christoph Räth, Claudia Scoccola, and Anthony Banday for useful discussions and comments, and Martin Reinecke, Mariapaola Bottino, and André Waelkens for their help with HEALPix. We acknowledge the use of the HEALPix package [7], the Legacy Archive for Microwave Background Data Analysis (LAMBDA), and CMBEASY [5].

\section{References}

[1] L. R. Abramo, A. Bernui, I. S. Ferreira, T. Villela, and C. A. Wuensche. Alignment tests for low CMB multipoles. Phys. Rev. D, 74(6):063506, September 2006.

[2] N. Afshordi. Integrated Sachs-Wolfe effect in cross-correlation: The observer's manual. Phys. Rev. D, 70(8):083536, October 2004.

[3] A. de Oliveira-Costa, M. Tegmark, M. Zaldarriaga, and A. Hamilton. Significance of the largest scale CMB fluctuations in WMAP. Phys. Rev. D, 69(6):063516, March 2004.

[4] K. Dolag, F. K. Hansen, M. Roncarelli, and L. Moscardini. The imprints of local superclusters on the Sunyaev-Zel'dovich signals and their detectability with Planck. MNRAS, 363:29-39, October 2005.

[5] M. Doran. CMBEASY: an object oriented code for the cosmic microwave background. Journal of Cosmology and Astro-Particle Physics, 10:11, October 2005.

[6] C. Dvorkin, H. V. Peiris, and W. Hu. Testable polarization predictions for models of CMB isotropy anomalies. Phys. Rev. D, 77(6):063008, March 2008.

[7] K. M. Górski, E. Hivon, A. J. Banday, B. D. Wandelt, F. K. Hansen, M. Reinecke, and M. Bartelmann. HEALPix: A Framework for High-Resolution Discretization and Fast Analysis of Data Distributed on the Sphere. ApJ, 622:759-771, April 2005.

[8] N. E. Groeneboom and H. K. Eriksen. Bayesian Analysis of Sparse Anisotropic Universe Models and Application to the Five-Year WMAP Data. ApJ, 690:1807-1819, January 2009. 
[9] G. Hinshaw et al. Five-Year Wilkinson Microwave Anisotropy Probe Observations: Data Processing, Sky Maps, and Basic Results. ApJS, 180:225-245, February 2009.

[10] K. T. Inoue and J. Silk. Local Voids as the Origin of Large-Angle Cosmic Microwave Background Anomalies: The Effect of a Cosmological Constant. ApJ, 664:650-659, August 2007.

[11] K. Land and J. Magueijo. The Axis of Evil revisited. MNRAS, 378:153-158, June 2007.

[12] M. Maturi, K. Dolag, A. Waelkens, V. Springel, and T. Enßlin. The actual Rees-Sciama effect from the local universe. $A \& A, 476: 83-88$, December 2007.

[13] J. A. Morales and D. Sáez. Large-Scale Vector Modes and the First CMB Temperature Multipoles. ApJ, 678:583-593, May 2008.

[14] L. Page et al. Three-Year Wilkinson Microwave Anisotropy Probe (WMAP) Observations: Polarization Analysis. ApJS, 170:335-376, June 2007.

[15] A. Rakić and D. J. Schwarz. Correlating anomalies of the microwave sky. Phys. Rev. D, 75(10):103002, May 2007.

[16] P. K. Samal, R. Saha, P. Jain, and J. P. Ralston. Testing isotropy of cosmic microwave background radiation. MNRAS, 385:1718-1728, April 2008.

[17] P. K. Samal, R. Saha, P. Jain, and J. P. Ralston. Signals of statistical anisotropy in WMAP foreground-cleaned maps. MNRAS, 396:511-522, June 2009.

[18] P. Vielva, Y. Wiaux, E. Martínez-González, and P. Vandergheynst. Alignment and signedintensity anomalies in Wilkinson Microwave Anisotropy Probe data. MNRAS, 381:932-942, November 2007. 